\title{
Formulation Development and Optimization of Oral Thin Films
}

\section{of Zolpidem Tartarate}

\author{
T.Neelima Rani ${ }^{1 *}$ \\ ${ }^{1}$ Mallareddy pharmacy college, Dhulapally, Secunderabad \\ *T.Neelima Rani, E-mail: neelimarani.tumma@gmail.com
}

Received: March 31, 2017

doi:10.22158/mshp.v1n1p26
Accepted: April 10, 2017

Online Published: April 16, 2017

URL: http://dx.doi.org/10.22158/mshp.v1n1p26

\begin{abstract}
The aim of the present study was to formulate and evaluate oral thin films of zolpidem tartarate. Zolpidem tartarateis used to treat insomnia. It affects chemicals in your brain that may become unbalanced and cause sleep problems (insomnia). Zolpidem tartarate oral thin films were prepared by using solvent casting method. In this method, water soluble polymer is completely dissolved in to form uniform clear viscous solution other ingredients including API are dissolved in a small portion of aqueous solvent by using a high shear processor. This viscous solution is degassed under the vacuum to remove the air bubbles. This bubble free solution is poured into a glass mold and kept in oven at 40 ${ }^{\circ}-50^{\circ} \mathrm{C}$. Oral disintegrating films are prepared using three grades of polymers HPMC E5, GUAR GUM and SODIUM ALGINATE Compatibility of Zolpidem tartarate with polymers was confirmed by FT-IR studies. All the formulations were evaluated for their physical appearance, average weight and thickness, folding endurance, disintegration time, tensile strength, percentage elongation, drug content, content uniformity and in vitro drug dissolution studies. From the result, it was concluded that the fast dissolving films of Zolpidem tartarate can be made by solvent casting technique with enhanced dissolution rate and taste masking by using suitable combination of sweeteners, flavors and citric acid. The final composition optimized was drug to Guar Gum ratio of 1:1, plasticizer concentration of 15\% w/w of polymer. The film had acceptable physical properties, assay and uniformity values and in vitro dissolution within 2 minutes.
\end{abstract}

\section{Keywords}

oral thin films, Zolpidem tartarate, solvent casting, placebo films, guar gum, sodium alginate, HPMC E5

\section{Introduction}

Fast-dissolving drug-delivery systems were first developed in the late 1970s as an alternative to tablets, capsules, and syrups for pediatric and geriatric patients who experience difficulties swallowing 
traditional oral solid-dosage forms. The novel technology of oral fast-dispersing dosage forms is known as fast dissolve, rapid dissolve, rapid melt and quick disintegrating tablets. However, the function and concept of all these dosage forms are similar. By definition, a solid dosage form that dissolves or disintegrates quickly in the oral cavity, resulting in solution or suspension without the need for the administration of water, is known as an oral fast-dispersing dosage form.

Oral films, also called oral wafers in the related literature, are a group of flat films which are administered into the oral cavity. Dissolvable Oral Thin Films (OTFs) or Oral Strip (OS) evolved over the past few years from the confection and oral care markets in the form of breath strips and became a novel and widely accepted form by consumers for delivering Vitamins and personal care products.

Many pharmaceutical companies have directed their research activity in reformulating existing drugs into new dosage forms. One such relatively new dosage form is the oral strip, a thin film that is prepared using hydrophilic polymers that rapidly dissolves on the tongue or buccal cavity. Developing formulations for children has been a challenging task. Amongst other factors, palatability of formulations of pediatric oral medications is one of the most Significant factors influencing compliance to therapeutic regimens.

Many pediatric and geriatric patients are unwilling to take these solid preparations due to fear of choking. Although oral disintegrating tablets have an advantage of administration without choking and fast disintegration; the disintegrated materials contained in them are insoluble and remain same until swallowing. In such cases formulation of fast dissolving film will be advantageous. So, in the present investigation we have prepared oral thin films of zolpidem tartarate. Oral thin films were prepared by using solvent casting, semisolid casting, hot melt extrusion, solid dispersion extrusion and rolling methods.

Zolpidem tartarate is a sedative, also called a hypnotic. Zolpidem tartarateis used to treat insomnia. It affects chemicals in your brain that may become unbalanced and cause sleep problems (insomnia). Zolpidem tartarate oral thin films were prepared by using solvent casting method. In this method, water soluble polymer is completely dissolved in to form uniform clear viscous solution other ingredients including API are dissolved in a small portion of aqueous solvent by using a high shear processor. This viscous solution is degassed under the vacuum to remove the air bubbles. This bubble free solution is poured into a glass mold and kept in oven at $40^{\circ}-50^{\circ} \mathrm{C}$.

\section{Materials and Methods}

Materials: Zolpidem Tartarate was obtained as a gift sample from EMCO industries, Hyderabad. HPMCE5, guar gum, sodium alginate are obtained from Loba chemicals, India. Glycerol, PEG 400, mannitol are obtained from Merck, India. All the solvents use was analytical grade.

\subsection{Methods}

\subsubsection{Construction of Calibration Curve}

Accurately weighed amount of $100 \mathrm{mg}$ of drug was dissolved in ethanol in $100 \mathrm{~mL}$ volumetric flask 
and the volume was made up to $100 \mathrm{~mL}$. This indicates the standard stock solution- $1 \mathrm{mg} / \mathrm{ml}$ in ethanol. From this stock solution $10 \mathrm{~mL}$ of solution was withdrawn and poured in to $100 \mathrm{~mL}$ volumetric flask and the volume was made up with phosphate buffer Ph 6.8 , this indicates the working stock-100 $\mu \mathrm{g} / \mathrm{ml}$. from this, working stock corresponding dilutions were made to get concentrations of 2, 4, 6, 8 and 10 $\mu \mathrm{g} / \mathrm{ml}$ and analyzed at $240 \mathrm{~nm}$ spectrophotometrically metrically against the reagent blank at $240 \mathrm{~nm}$ and a calibration curve was constructed.

\subsubsection{Drug Polymer Compatibility Studies}

The infrared spectra of drug, polymers, physical mixture of drug and polymers were recorded at a scanning range of 400-4000 $\mathrm{cm}^{-1}$ using FTIR spectrometer.

\subsection{Formulation Development}

\subsubsection{Placebo Formulations}

Placebo formulations were prepared by using solvent casting technique. The film forming polymers HPMCE5, GUAR GUM and SODIUM ALGINATE were accurately weighed and dispersed in $10 \mathrm{ml}$ of distilled water each and then soaked for 4 hours. To these polymer solutions, Mannitol was added with continuous stirring on a magnetic stirrer. The resulting bubble free solution was poured on to a glass plate and was dried at $50^{\circ} \mathrm{C}$ in hot air oven for $24 \mathrm{hr}$. The placebo films formed were carefully removed from glass plate and evaluated for drying time, average weight, thickness, tensile strength, folding endurance and in vitro disintegration time. The placebo film formulations are given in Table 1 and Table 2.

\subsubsection{Drug Loaded Formulations}

The composition for the drug loaded films is given in Table 3. The procedure followed was similar to the placebo formulations. The Zolpidem Tartarate was added to the polymer solution and sonicated for 15 minutes till the drug was completely dissolved. The drug loaded films were evaluated for average weight, thickness, tensile strength, folding endurance, in vitro disintegration time and in vitro dissolution studies.

Table 1. Composition of Placebo Formulations with Glycerol as Plasticizer

\begin{tabular}{|c|c|c|c|c|c|c|c|c|c|c|c|}
\hline Ingredients & $5 f 1$ & $5 f 2$ & $5 f 3$ & $5 f 4$ & $15 f 1$ & $15 f 2$ & $15 f 3$ & $15 f 4$ & Pf1 & Pf2 & Pf3 \\
\hline HPMC E5 (mg) & 62.5 & 125 & 187.5 & 250 & - & - & - & - & - & - & - \\
\hline GUAR GUM (mg) & - & - & - & - & 62.5 & 125 & 187.5 & 250 & - & - & - \\
\hline $\begin{array}{l}\text { SODIUM } \\
\text { ALGINATE (mg) }\end{array}$ & - & - & - & - & - & - & - & - & 125 & 187.5 & 250 \\
\hline GLYCEROL (mg) & 9.38 & 18 & 28.1 & 37.5 & 9.38 & 18.7 & 28.1 & 37.5 & 18.7 & 28.1 & 37.5 \\
\hline MANNITOL (mg) & 100 & 100 & 100 & 100 & 100 & 100 & 100 & 100 & 100 & 100 & 100 \\
\hline
\end{tabular}




\begin{tabular}{llllllllllll}
\hline DISTILLED & 10 & 10 & 10 & 10 & 10 & 10 & 10 & 10 & 10 & 10 & 10 \\
WATER $(\mathrm{ml})$ & & & & & & & & & &
\end{tabular}

Table 2. Composition of Placebo Formulations with PEG 400 as Plasticizer

\begin{tabular}{|c|c|c|c|c|c|c|c|c|c|c|c|}
\hline INGREDIENTS & $5 f 5$ & $5 f 6$ & $\mathbf{5 f 7}$ & $5 f 8$ & $15 f 5$ & $15 f 6$ & $15 f 7$ & $15 f 8$ & Pf4 & Pf5 & Pf6 \\
\hline HPMC E5 (mg) & 62.5 & 125 & 187.5 & 250 & - & - & - & - & - & - & - \\
\hline GUAR GUM (mg) & - & - & - & - & 62.5 & 125 & 187.5 & 250 & - & - & - \\
\hline SODIUM & & & & & & & & & & & \\
\hline ALGINATE (mg) & - & - & - & - & - & - & - & - & 125 & 187.5 & 250 \\
\hline PEG 400 (mg) & 9.38 & 18 & 28.1 & 37.5 & 9.38 & 18.7 & 28.1 & 37.5 & 18.7 & 28.1 & 37.5 \\
\hline MANNITOL (mg) & 100 & 100 & 100 & 100 & 100 & 100 & 100 & 100 & 100 & 100 & 100 \\
\hline $\begin{array}{l}\text { DISTILLED WATER } \\
\text { (ml) }\end{array}$ & 10 & 10 & 10 & 10 & 10 & 10 & 10 & 10 & 10 & 10 & 10 \\
\hline
\end{tabular}

Table 3. Composition of Drug Loaded Zolpidem Tartarate Films

\begin{tabular}{|c|c|c|c|c|c|c|c|}
\hline \multirow[b]{2}{*}{ Ingredients } & \multicolumn{3}{|c|}{ HPMC E5 } & \multicolumn{4}{|c|}{ GUAR GUM } \\
\hline & F1 & F2 & F3 & F4 & F5 & F6 & F7 \\
\hline Drug (mg) & 62.5 & 62.5 & 62.5 & 62.5 & 62.5 & 62.5 & 62.5 \\
\hline Polymer (mg) & 125 & 187.5 & 250 & 62.5 & 125 & 187.5 & 250 \\
\hline Glycerol (mg) & 18.7 & 28.1 & 37.5 & 9.38 & 18.7 & 28.1 & 37.5 \\
\hline Mannitol (mg) & 100 & 100 & 100 & 100 & 100 & 100 & 100 \\
\hline Flavour & $5 \%$ & $5 \%$ & $5 \%$ & $5 \%$ & $5 \%$ & $5 \%$ & $5 \%$ \\
\hline Distilled Water(ml) & 10 & 10 & 10 & 10 & 10 & 10 & 10 \\
\hline
\end{tabular}

\section{Evaluation of the Oral Dissolving Films}

\subsection{Average Weight Measurements}

The weight of the films was determined by Analytical Balance. One centimeter square was cut at five different places in the casted film. The weight of each film was taken and the average weight was calculated. Uniformity in weight of the films indicates accuracy of dose of the oral films.

\subsection{Thickness}

Uniformity in the thickness of the film was related in accuracy of dose of the ODFs. At different locations of the strip the thickness was measured using Vernier Calipers Micrometer. Folding Endurance: Folding endurance indicates mechanical strength of the oral films and was determined by folding the strip repeatedly at the same place till it breaks. The number of times the film was folded without breaking and it was computed as the folding endurance value. 


\subsection{Tensile Strength}

Tensile strength indicates mechanical strength of the oral films. It indicates maximum stress required to break the strip when applied at a point. It was determined using Texture Analyzer Stable Micro system.

\subsection{Percentage Elongation}

Percentage elongation of the strip was determined using Texture Analyzer Stable Micro system. The strip was taken and force was gradually applied till the film elongates and finally breaks. The readings were taken from the instrument.

\subsection{Assay and Content Uniformity}

Films of $2 \times 1 \mathrm{~cm}^{2}$ were taken and dissolved in $\mathrm{pH} 6.8$ Phosphate buffer and made up to $100 \mathrm{ml}$ in a volumetric flask. Then $1 \mathrm{~mL}$ was withdrawn from the solution and diluted to $10 \mathrm{~mL}$. The absorbance of the solution was measured spectrophotometrically at $240 \mathrm{~nm}$. The assay was calculated by this method. Limit of content uniformity is $85-115 \%$.

\subsection{Disintegration Time}

The film equivalent to one dose $\left(2 \times 1 \mathrm{~cm}^{2}\right)$ was placed in a glass petridish containing $10 \mathrm{ml}$ of $\mathrm{pH} 6.8$ phosphate buffer. The time required for the film to break was noted as disintegration time.

\subsection{In Vitro Dissolution Studies}

The in-vitro dissolution studies were conducted using simulated saliva $(500 \mathrm{~mL})$. The dissolution studies were carried out using USP dissolution apparatus I (Electrolab, Mumbai, India) at $37 \pm 0.5{ }^{\circ} \mathrm{C}$ and at $50 \mathrm{rpm}$ using specified dissolution media. Each film with dimension $\left(2 \times 1 \mathrm{~cm}^{2}\right)$ was placed on a stainless-steel wire mesh. The film sample placed on the sieve was submerged into dissolution media. Samples were withdrawn at $0,2,5,10,15$ and 30 min. time intervals and filtered through wattmann filter paper and were analyzed spectrophotometrically at $240 \mathrm{~nm}$. To maintain the volume, an equal volume of fresh dissolution medium maintained at same temperature was added after withdrawing samples. The absorbance values were converted to concentration using standard calibration curve previously obtained by experiment.

\section{Kinetic Modelling}

\subsection{Release Kinetic Models}

Model dependent methods are based on different mathematical functions, which describe the dissolution profile. Once a suitable function has been selected, the dissolution profiles can easily be evaluated depending on the derived model parameters. The model dependent approaches includes zero order, first order, Higuchi, Hixson-Crowell, Korsmeyer-Peppas, Baker-Lonsdale, Weibull, Hopfenberg, Gompertz, Non-conventional order 1, Non-conventional order 2, Reciprocal powered time and regression models. Among them tried four models to fit the data with zero order, first order, Higuchi, and Korsmeyer-Peppas models. 


\subsection{Zero-Order Model}

Drug dissolution from dosage forms that do not disaggregate and release the drug slowly can be represented by the equation:

$Q_{0}-Q_{T}=k_{0}{ }^{T} \quad$ Rearrangement of equation yields:

$Q_{T}=Q_{0}+k_{0}{ }^{T}$

Where $\mathrm{Q}_{t}$ is the amount of drug dissolved in time $t, \mathrm{Q}_{0}$ is the initial amount of drug in the solution (most of the times, $\mathrm{Q}_{0}=0$ ) and $\mathrm{K}_{0}$ is the zero order release constant expressed in units of concentration/time.

Plotted the cumulative amount of drug released versus time. This relationship can be used to describe the drug dissolution of several types of modified release pharmaceutical dosage forms as in the case of some transdermal systems, as well as matrix tablets with low soluble drugs, coated forms, osmotic systems, etc.

\subsection{First Order Model}

The application of this model to drug dissolution studies was first proposed by Gibaldi and Feldman (1967) and later by Wagner (1969). This model has been also used to describe absorption and/or elimination of some drugs, although it is difficult to conceptualize this mechanism on a theoretical basis. The release of the drug which follows first order kinetics can be expressed by the equation:

$\frac{d_{c}}{d_{t}}=-k_{c}$

Where $\mathrm{K}$ is first order rate constant expressed in units of time ${ }^{-1}$.

Equation can be expressed in log form as:

$\log C=\log C_{0}-\frac{k_{t}}{2.303}$

Where $\mathrm{C}_{0}$ is the initial concentration of drug, $\mathrm{K}$ is the first order rate constant, and $\mathrm{t}$ is the time. The data obtained are plotted as log cumulative percentage of drug remaining vs. time which would yield a straight line with a slope of $-\mathrm{K} / 2.303$.

\subsection{Higuchi Model}

This model is used to study the release of water soluble and poorly soluble drugs incorporated in semi-solid and/or solid matrices. Mathematical expressions are obtained for drug particles dispersed in a uniform matrix behaving as the diffusion media. To study the dissolution from a planar system having a homogeneous matrix, the relation obtained is as following:

$$
f_{t}=Q=\sqrt{D\left(2 C-C_{s}\right) C_{s} t}
$$

Where Qis the amount of drug released in time tper unit area, Cis the drug initial concentration, Cis the drug $S$ solubility in the matrix media and Dis the diffusivity of the drug molecules (diffusion constant) in the matrix substance. This relation was first proposed by Higuchi $(1961,1963)$ to describe the dissolution of drugs in suspension from ointments bases, but is clearly in accordance with other types of dissolution from other pharmaceutical dosage forms. 
In a general way it is possible to resume the Higuchi model to the following expression (generally known as the simplified Higuchi model):

$f_{t}=K_{H} t^{\frac{1}{2}}$

Where $K_{H}$ is the Higuchi dissolution constant treated sometimes in a different manner by different authors and theories. Higuchi describes drug release as a diffusion process based in the Fick's law, square root time dependent. This relation can be used to describe the drug dissolution from several types of modified release pharmaceutical dosage forms, as in the case of some trans dermal systems and matrix tablets with water soluble drugs.

\section{Results and Discussion}

\subsection{Preformulation Studies}

Calibration curve of Zolpidem tartarate: The absorbance values for different concentration of Zolpidem tartarate standard solutions are recorded in Table 4 and shown in Figure 1.

Table 4. Calibration Curve of Zolpidem Tartarate in Phosphate Buffer pH 6.8

\begin{tabular}{ll}
\hline Concentration $(\boldsymbol{\mu g} / \mathbf{m l})$ & Absorbance at $\mathbf{2 4 0} \mathbf{~ n m}$ \\
\hline 0 & 0 \\
2 & 0.172 \\
4 & 0.325 \\
6 & 0.501 \\
8 & 0.683 \\
10 & 0.829 \\
\hline
\end{tabular}

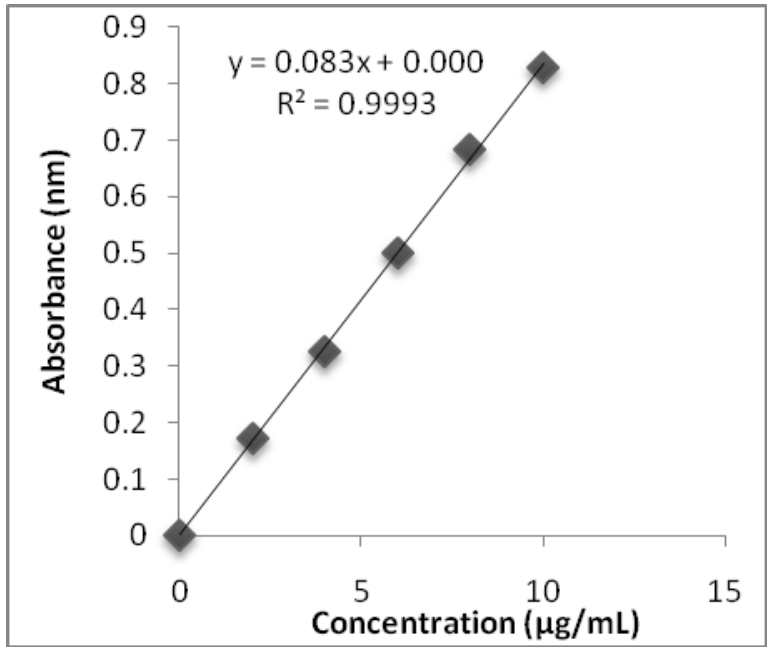

Figure 1. Calibration Curve of Zolpidem Tartarate in Phosphate Buffer pH 6.8 
Figure 1 indicates the UV-spectrometric method is linear in the range of 2 to $10 \mathrm{ug} / \mathrm{ml}$ and obtained the $\mathrm{R}^{2}$ value as 0.9993 . From the equation, we can calculate the concentration of the samples measured.

\subsection{Drug-Excipients Compatibility Studies}

FTIR spectrum of the drug and drug in the presence of the polymers was recorded and the characteristic peaks of the drug identified in the physical mixture indicating the absence of the interaction of the drug with polymers. As shown in Table 5, the characteristic peaks of the drug for the bonds occur at similar wave numbers with same intensity in the physical mixture and pure drug.

Table 5. FTIR Interpretation of Zolpidem Tartarate and Guar Gum

\begin{tabular}{llll}
\hline Functional groups & Zolpidem tartarate & Guar Gum & Formulation \\
\hline O-H Stretching & $3738 \mathrm{~cm}^{-1}$ & $3755 \mathrm{~cm}^{-1}$ & $3744 \mathrm{~cm}^{-1}$ \\
N-H stretching & $3398 \mathrm{~cm}^{-1}$ & - & $3393 \mathrm{~cm}^{-1}$ \\
C=O stretching & $1656 \mathrm{~cm}^{-1}$ & - & $1633 \mathrm{~cm}^{-1}$ \\
C-H stretching & $2900 \mathrm{~cm}^{-1}$ & $2910 \mathrm{~cm}^{-1}$ & $2939 \mathrm{~cm}^{-1}$ \\
C=N Stretching & $1508 \mathrm{~cm}^{-1}$ & - & $1509 \mathrm{~cm}^{-1}$ \\
\hline
\end{tabular}

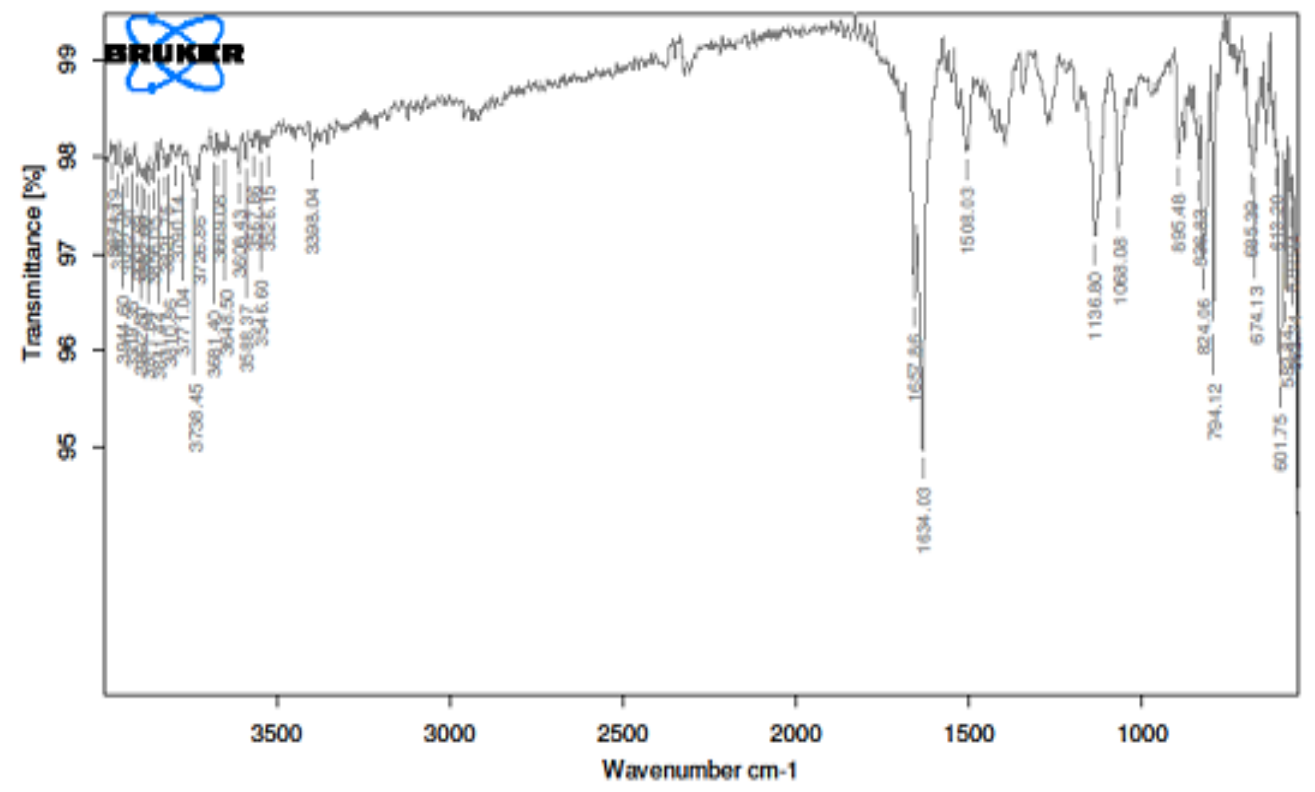

Figure 2. FTIR of Zolpidem Tartarate 


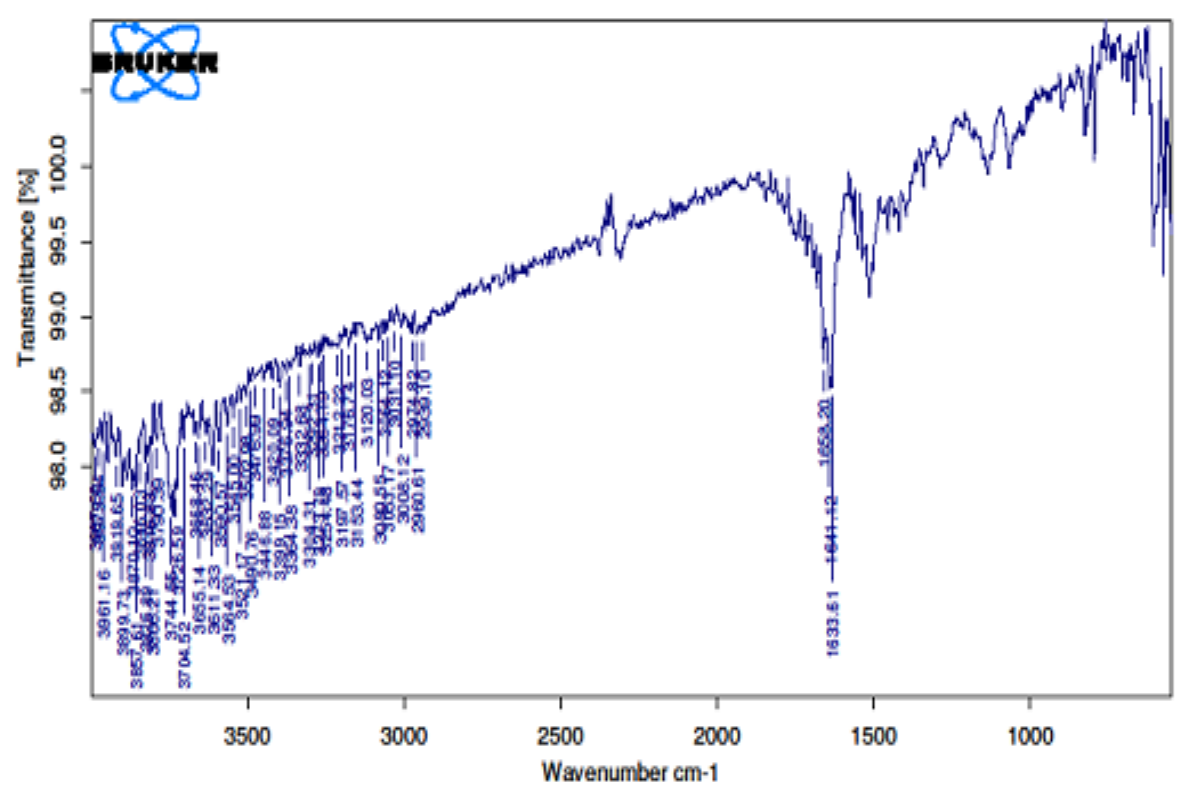

Figure 3. FTIR of Formulation

The characteristic peaks of pure drug, GUAR GUM, physical mixture were illustrated in Figures 2, 3. The O-H stretching, $\mathrm{N}-\mathrm{H}$ stretching, $\mathrm{C}=\mathrm{O}$ stretching, $\mathrm{C}-\mathrm{H}$ stretching and heterocyclic $\mathrm{C}=\mathrm{N}$ stretching of zolpidem tartarate was observed at $3738 \mathrm{~cm}^{-1}, 3398 \mathrm{~cm}^{-1}, 1656 \mathrm{~cm}^{-1}, 2900 \mathrm{~cm}^{-1}$ and $1508 \mathrm{~cm}^{-1}$ respectively. The $\mathrm{O}-\mathrm{H}$ stretching, $\mathrm{N}-\mathrm{H}$ stretching, $\mathrm{C}=\mathrm{O}$ stretching, $\mathrm{C}-\mathrm{H}$ stretching and heterocyclic $\mathrm{C}=\mathrm{N}$ stretching of the formulation was observed at $3744 \mathrm{~cm}^{-1}, 3393 \mathrm{~cm}^{-1}, 1633 \mathrm{~cm}^{-1}, 2939 \mathrm{~cm}^{-1}$ and $1509 \mathrm{~cm}^{-1}$. From this it can be concluded that there is no change in the $\mathrm{O}-\mathrm{H}$ stretching, N-H stretching, $\mathrm{C}=\mathrm{O}$ stretching and $\mathrm{C}=\mathrm{N}$ stretching of the drug and the formulation. There is a slight but not significant change in the $\mathrm{C}-\mathrm{H}$ stretching of the formulation than the drug. So, in the formulation, no considerable changes were observed in the peaks and it can be concluded that there are no interactions with the drug and the peak height, intensity, position of peak of drug is similar without variations indicating that drug and polymers are compatible. This interaction study revealed that no toxic effects can be found in the formulation.

\section{Evaluation of Oral Dissolving Films}

\subsection{Placebo Films}

The plasticizers used were PEG 400 and Glycerol. Films made by PEG 400 were sticky and they are difficult to remove from the plate. Plasticity and tackiness is also poor for these films. So, glycerol was selected as an optimized plasticizer. Concentration of glycerol less than $15 \% \mathrm{w} / \mathrm{w}$ was not enough to plasticize the films and therefore $15 \% \mathrm{w} / \mathrm{w}$ of the glycerol was selected as an optimized concentration. The drying time for Sodium Alginate based films were unacceptably long ( $>24$ hours). Also these films were much inferior to HPMC based films in terms disintegration time and thickness values. The HPMC E5 and guar gum films showed acceptable values for the evaluated parameters and were selected for 
the drug loading studies. Among the films made of HPMC E5 5f1 (1:1) formulation showed poor results. So, it is not selected for drug loading studies.

Table 6. Evaluation Parameters of HPMC E5, GUAR GUM and SODIUM ALGINATE

\begin{tabular}{|c|c|c|c|c|c|c|c|c|}
\hline \multirow{2}{*}{ Evaluation parameters } & \multicolumn{3}{|c|}{ HPMC E 5} & \multicolumn{5}{|c|}{ GUAR GUM } \\
\hline & $5 \mathrm{f} 1$ & $5 f 2$ & $5 \mathrm{f} 3$ & $5 f 4$ & $15 \mathrm{f} 1$ & $15 f 2$ & $15 \mathrm{f} 3$ & $15 f 4$ \\
\hline Drying time (hrs) & $16-24$ & $16-24$ & $16-24$ & $16-24$ & $16-24$ & $16-24$ & $16-24$ & $16-24$ \\
\hline $\begin{array}{l}\text { in vitro Disintegration } \\
\text { time (secs) }\end{array}$ & $22 \pm 5$ & $52 \pm 2$ & $59 \pm 3$ & $63 \pm 2$ & $47 \pm 3$ & $58 \pm 1$ & $66 \pm 2$ & $77 \pm 2$ \\
\hline Folding endurance & $28 \pm 4$ & $119 \pm 1$ & $129 \pm 2$ & $134 \pm 1$ & $110 \pm 3$ & $122 \pm 3$ & $133 \pm 1$ & $140 \pm 1$ \\
\hline Average weight (mg) & $4 \pm 3$ & $10 \pm 4$ & $14 \pm 3$ & $18 \pm 2$ & $8.5 \pm 3$ & $14 \pm 3$ & $17 \pm 2$ & $20 \pm 1$ \\
\hline Thickness (mm) & $0.09 \pm 0.2$ & $0.07 \pm 0.1$ & $\begin{array}{l}0.1 \pm \\
0.01\end{array}$ & $0.121 \pm 0.02$ & $0.06 \pm 0.01$ & $0.105 \pm 0.01$ & $0.12 \pm 0.2$ & $0.14 \pm 0.1$ \\
\hline $\begin{array}{l}\text { Tensile } \quad \text { strength } \\
\left(\mathbf{N} / \mathbf{c m}^{2}\right)\end{array}$ & $0.02 \pm 0.4$ & $0.99 \pm 0.5$ & $1.65 \pm 0.1$ & $1.99 \pm 0.02$ & $1.74 \pm 0.03$ & $1.99 \pm 0.1$ & $2.28 \pm 0.2$ & $2.78 \pm 0.2$ \\
\hline \multirow{2}{*}{\multicolumn{3}{|c|}{ Evaluation parameters }} & \multicolumn{4}{|c|}{ SODIUM ALGINATE } & & \\
\hline & & & \multicolumn{2}{|c|}{ Pf1 } & \multicolumn{2}{|l|}{$\operatorname{Pf} 2$} & \multicolumn{2}{|l|}{ Pf3 } \\
\hline \multicolumn{3}{|l|}{ Drying time(hrs) } & \multicolumn{2}{|c|}{$>24$} & \multicolumn{2}{|l|}{$>24$} & \multicolumn{2}{|l|}{$>24$} \\
\hline \multicolumn{3}{|c|}{ in vitro Disintegration time (secs) } & \multicolumn{2}{|c|}{$130 \pm 2$} & \multicolumn{2}{|c|}{$152 \pm 1$} & \multicolumn{2}{|l|}{$179 \pm 3$} \\
\hline \multicolumn{3}{|l|}{ Folding endurance } & \multicolumn{2}{|c|}{$162 \pm 4$} & \multicolumn{2}{|c|}{$184 \pm 2$} & \multicolumn{2}{|l|}{$191 \pm 5$} \\
\hline \multicolumn{3}{|l|}{ Average weight(mg) } & \multicolumn{2}{|c|}{$48 \pm 4$} & \multicolumn{2}{|l|}{$61 \pm 3$} & \multicolumn{2}{|l|}{$70 \pm 2$} \\
\hline \multicolumn{3}{|l|}{ Thickness (mm) } & \multicolumn{2}{|c|}{$0.182 \pm 0.02$} & \multicolumn{2}{|c|}{$0.206 \pm 0.01$} & \multicolumn{2}{|c|}{$0.311 \pm 0.02$} \\
\hline \multicolumn{3}{|c|}{ Tensile strength $\left(\mathrm{N} / \mathrm{cm}^{2}\right)$} & \multicolumn{2}{|c|}{$3.78 \pm 0.01$} & \multicolumn{2}{|c|}{$4.03 \pm 0.03$} & \multicolumn{2}{|c|}{$4.97 \pm 0.02$} \\
\hline
\end{tabular}

\section{Drug Loaded Films}

\subsection{Physical Appearance of the Films}

The pictures of ODFs of all the formulations are shown in Figure 4. The pictures A, B and C indicates HPMC E 5 containing films, it was observed that the films were thin with lower peal ability, though they were transparent. The viscosity of the film former was 5 cps (Rowe et al., 2006) which was lower resulting in thin films. The pictures E, F, G and $\mathrm{H}$ indicate Guar Gum containing films. It was observed that the films with Guar Gum were transparent with good peal ability, flexibility and with smooth surface when compared to HPMC E 5 containing films. This is because the viscosity of the film former being high (Guar Gum) (Rowe et al., 2006). The results were comparable with reports of Kaur and Bala (2012) where the effect of physical appearance of the films were studied in the presence of Guar Gum containing films and with Vijayasri (2012) where the promising polymer Guar Gum is showing good 
physical characteristics.

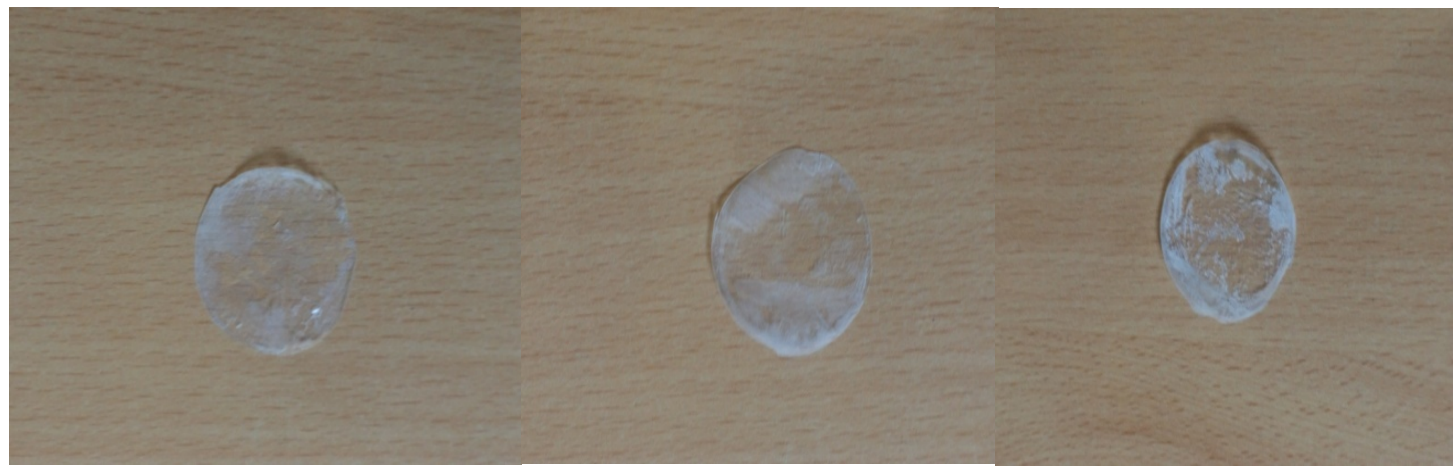

A

B

$\mathrm{C}$

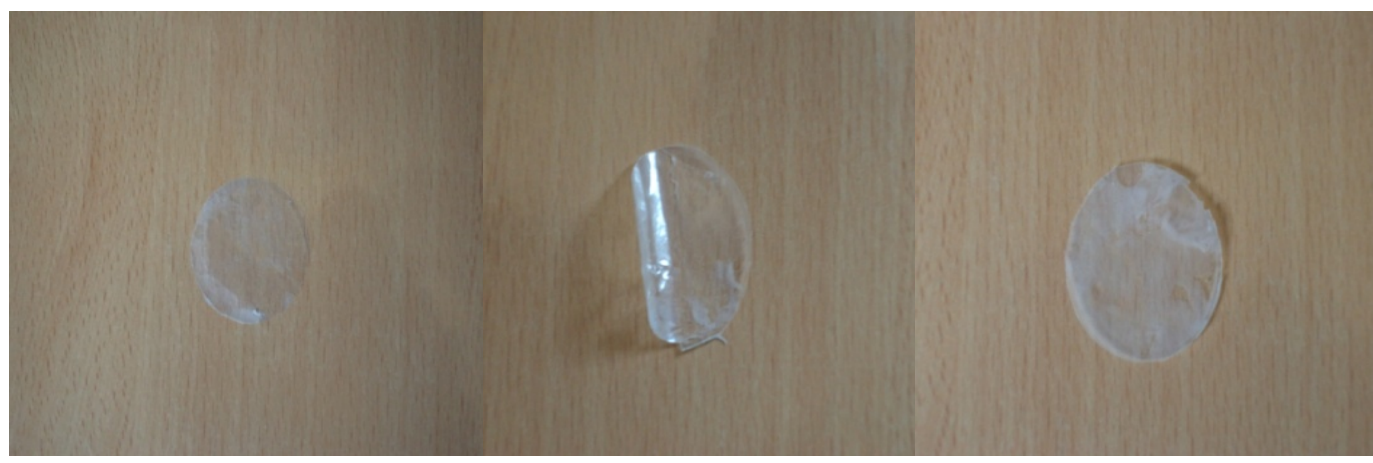

E

F

G

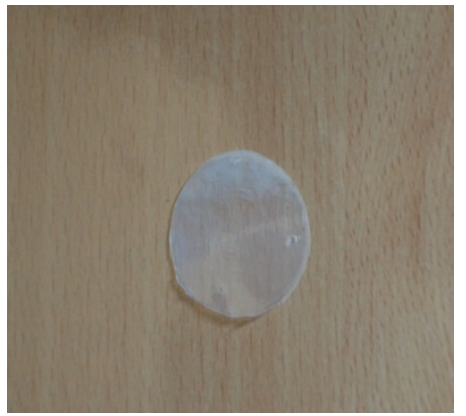

Figure 4. Physical Appearance of Drug Loaded Formulations

Table 7. Comparative Results of Evaluation Parameters of ODF Formulations

\begin{tabular}{|c|c|c|c|c|c|c|c|c|}
\hline $\begin{array}{l}\text { Formulation } \\
\text { code }\end{array}$ & $\begin{array}{l}\text { Average } \\
\text { weight } \\
\text { (mg) }\end{array}$ & $\begin{array}{l}\text { Thickness } \\
\text { (mm) }\end{array}$ & $\begin{array}{l}\text { Folding } \\
\text { endurance }\end{array}$ & $\begin{array}{l}\text { invitro } \\
\text { disintegration } \\
\text { time (secs) }\end{array}$ & $\begin{array}{l}\text { Tensile } \\
\text { strength } \\
\left(\mathrm{N} / \mathrm{cm}^{2}\right)\end{array}$ & $\begin{array}{l}\% \\
\text { elongation }\end{array}$ & $\begin{array}{l}\text { Drug } \\
\text { content } \\
\text { (mg) }\end{array}$ & $\begin{array}{l}\text { Content } \\
\text { uniformity } \\
(\%)\end{array}$ \\
\hline F1 & $16.5 \pm 0.3$ & $0.082 \pm 0.01$ & $119 \pm 1$ & $52 \pm 1$ & $1.03 \pm 0.05$ & $25.44 \pm 0.68$ & 3.52 & $103-105$ \\
\hline F2 & $18 \pm 0.4$ & $0.109 \pm 0.02$ & $130.3 \pm 9$ & $60 \pm 2$ & $1.87 \pm 0.01$ & $49.5 \pm 0.62$ & 3.48 & 89-101 \\
\hline
\end{tabular}




\begin{tabular}{|c|c|c|c|c|c|c|c|c|}
\hline F3 & $19.9 \pm 0.8$ & $0.126 \pm 0.01$ & $136.3 \pm 3$ & $65 \pm 2$ & $2.23 \pm 0.04$ & $65.5 \pm 0.50$ & 3.39 & $83-91$ \\
\hline F4 & $14.7 \pm 0.7$ & $0.081 \pm 0.02$ & $112 \pm 3$ & $49 \pm 2$ & $1.88 \pm 0.02$ & $25.62 \pm 0.82$ & 3.57 & $100-104$ \\
\hline F5 & $19 \pm 2.6$ & $0.113 \pm 0.01$ & $134 \pm 2$ & $58 \pm 1$ & $2.03 \pm 0.07$ & $38.94 \pm 0.83$ & 3.60 & $85-93$ \\
\hline F6 & $21.4 \pm 0.4$ & $0.134 \pm 0.05$ & $145.3 \pm 2$ & $69 \pm 2$ & $2.43 \pm 0.02$ & $69.2 \pm 0.36$ & 3.46 & $85-87$ \\
\hline
\end{tabular}

\subsection{In Vitro Dissolution Studies of ODFs}

The in vitro drug dissolution study of Zolpidem tartarate from all the formulations was performed by using USP Type I basket apparatus, using $500 \mathrm{~mL}$ of phosphate buffer $\mathrm{pH} 6.8$.

Drug release from formulations $F 1, F 2$ and F3: F1, F2 and F3 formulations are formulated using HPMC E5 as film former at 1:2, 1:3 and 1:4 ratios of drug to polymer respectively. Percent drug release profiles from formulations F1, F2 and F3 are shown in Figure 5 and the Table 8. As the ODFs are thin films with more surface area, they get wet quickly. Within 15 minutes $99.6 \%$ of the drug was released from $\mathrm{F} 1$ formulation, $96.2 \%$ of the drug from $\mathrm{F} 2$ formulation and 94.9 from $\mathrm{F} 3$ formulation. As the film former concentration was increased the rate of drug release was decreased because of the decreased rate of erosion of the films.

Table 8. Drug Release Profiles of Formulations with Film Former HPMC E5-F1, F2 and F3

\begin{tabular}{llll}
\hline Time (mins) & F1 & F2 & F3 \\
\hline 0 & 0 & 0 & 0 \\
2 & $88 \pm 0.01$ & $81.5 \pm 0.01$ & $76.4 \pm 0.05$ \\
5 & $92 \pm 0.11$ & $89 \pm 0.03$ & $84.6 \pm 0.12$ \\
10 & $97.28 \pm 0.20$ & $94.6 \pm 0.05$ & $89.6 \pm 0.15$ \\
15 & $99.6 \pm 0.02$ & $96.2 \pm 0.11$ & $94.9 \pm 0.20$ \\
30 & $100 \pm 0.10$ & $97 \pm 0.12$ & $95.8 \pm 0.02$ \\
\hline
\end{tabular}




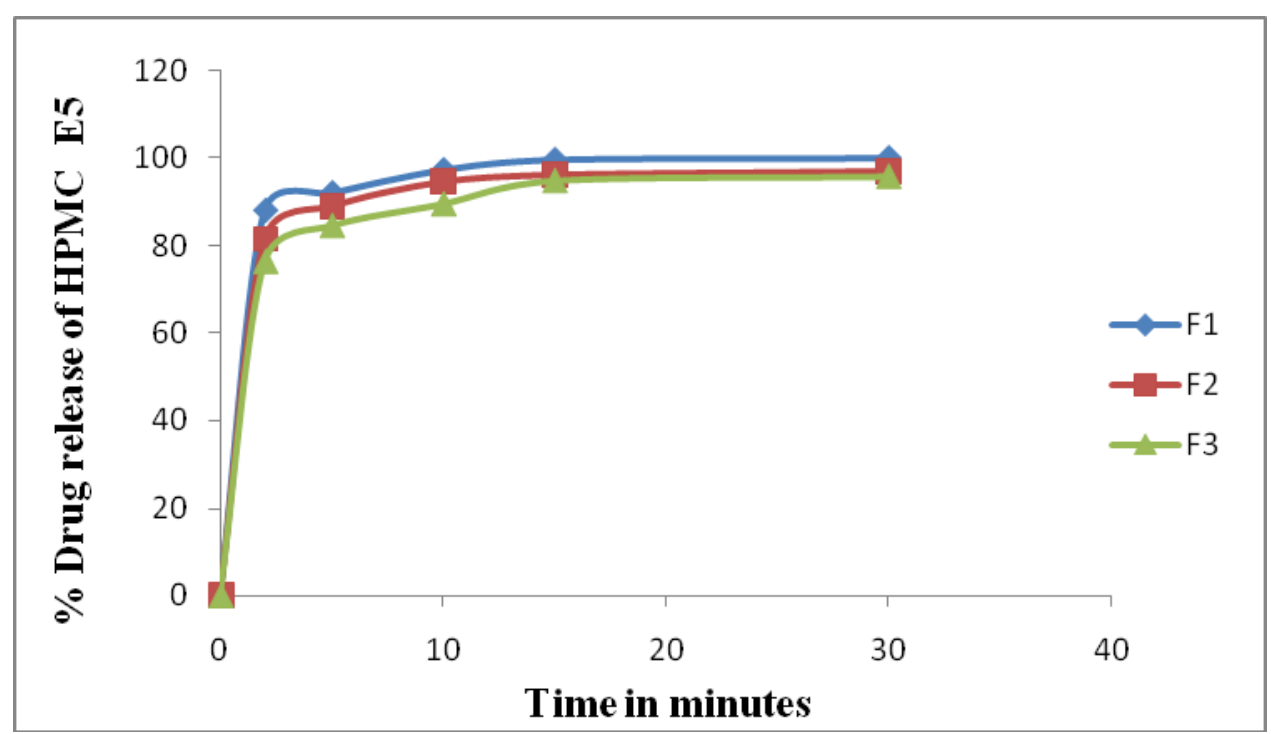

Figure 5. Comparative Percent Drug Release Profiles from Formulations F1, F2 and F3

Drug release from formulations F4, F5, F6 and F7: F4, F5, F6 and F7 formulations are formulated using Guar Gum as film former at 1:1, 1:2, 1:3 and 1:4 ratios of drug to polymer respectively. From the Figure 6 and the Table 9, it can be observed that the F4 formulation showed faster release of the drug about 99.98 within 15 minutes than F5, F6 and F7, where the percent drug release was 98.4, 94.7 and 92.9 respectively. As the film former concentration was increased the rate of drug release was decreased because the erosion of the films might have probably delayed.

Table 9. Drug Release Profiles of Formulations with Former Guar Gum-F4, F5, F6 and F7

\begin{tabular}{lllll}
\hline Time (mins) & F4 & F5 & F6 & F7 \\
\hline 0 & 0 & 0 & 0 & 0 \\
2 & $89.2 \pm 0.10$ & $85 \pm 0.05$ & $80.9 \pm 0.05$ & $76 \pm 0.05$ \\
5 & $94 \pm 0.06$ & $90 \pm 0.08$ & $88.91 \pm 0.01$ & $84 \pm 0.08$ \\
10 & $98.81 \pm 0.11$ & $94 \pm 0.10$ & $92.7 \pm 0.06$ & $86.8 \pm 0.11$ \\
15 & $99.98 \pm 0.05$ & $98.4 \pm 0.11$ & $94.7 \pm 0.14$ & $92.9 \pm 0.10$ \\
30 & $101.81 \pm 0.11$ & $99.6 \pm 0.12$ & $95 \pm 0.11$ & $93 \pm 0.13$ \\
\hline
\end{tabular}




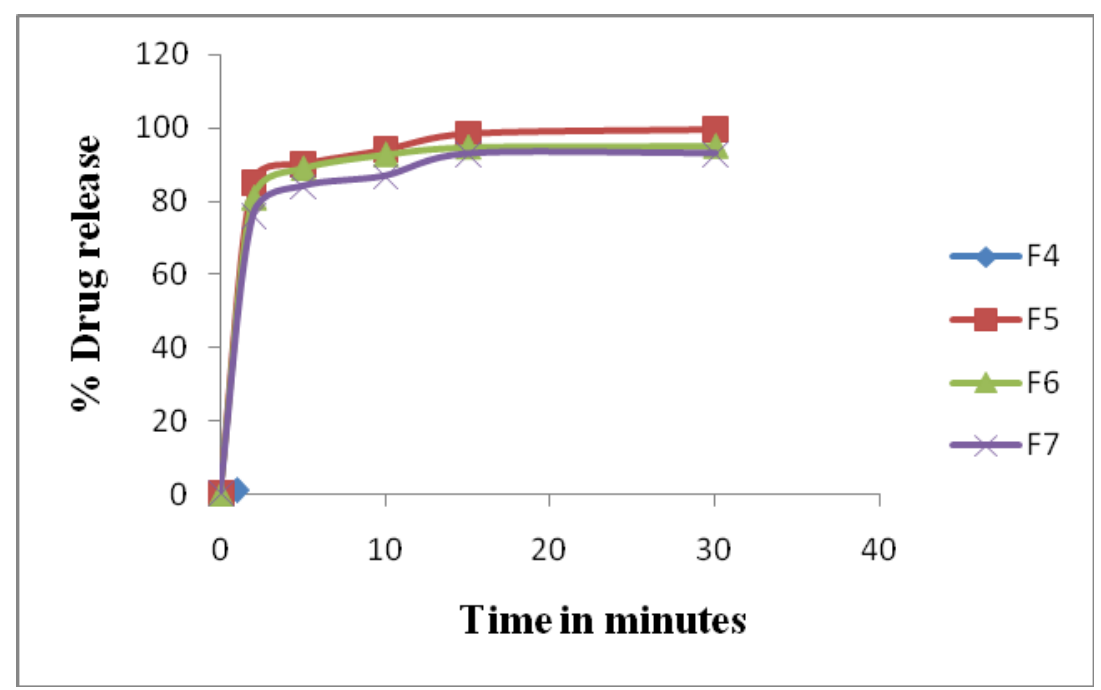

Figure 6. Comparative Drug Release Profiles from Formulations F4, F5, F6 and F7

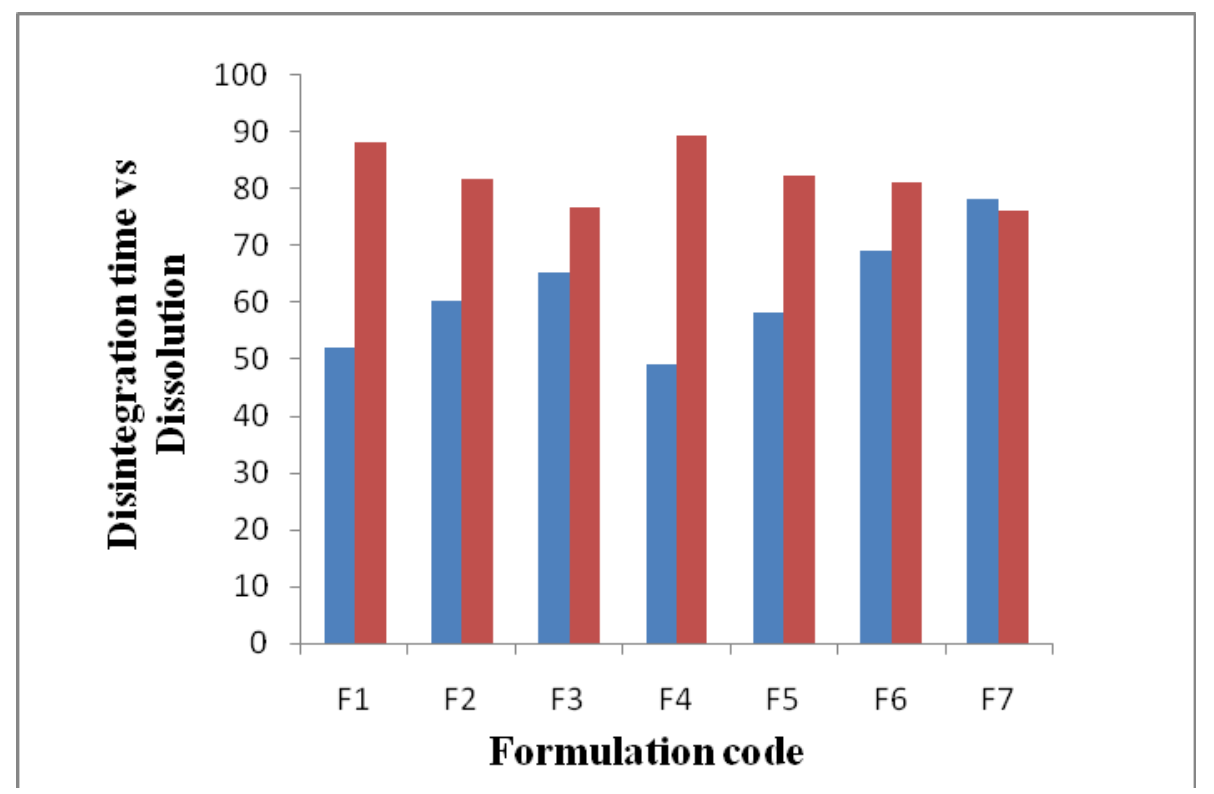

Figure 7. Comparison of Disintegration Time and Drug Release in 2 Minutes of ODFs

There is a marginal but not significant difference in dissolution between different compositions. The dissolution profile (\% Drug release in 2 mins) Vs disintegration time (seconds) is shown in Figure 7. 


\subsection{Release Kinetics}

Table 10. Release Rate Kinetics

\begin{tabular}{lllllll}
\hline \multirow{2}{*}{ Formulation Code } & \multicolumn{2}{c}{ Zero Order Equation } & \multicolumn{2}{c}{ First Order Equation } & \multicolumn{2}{c}{ Higuchi Equation } \\
\cline { 2 - 7 } & K & $\mathbf{R}^{\mathbf{2}}$ & $\mathbf{K}$ & $\mathbf{R}^{\mathbf{2}}$ & $\mathbf{N}$ & $\mathbf{R}^{\mathbf{2}}$ \\
\hline F1 & 0.8955 & 0.9294 & 0.1385 & 0.9573 & 81.266 & 0.9916 \\
F2 & 1.087 & 0.8032 & 0.0818 & 0.8756 & 74.219 & 0.9631 \\
F3 & 1.3398 & 0.8341 & 0.0721 & 0.8453 & 66.936 & 0.985 \\
F4 & 0.8192 & 0.9642 & 0.2197 & 0.9902 & 83.45 & 0.9951 \\
F5 & 0.9878 & 0.8776 & 0.0968 & 0.9013 & 77.667 & 0.9483 \\
F6 & 0.9813 & 0.7413 & 0.0708 & 0.8469 & 74.698 & 0.9267 \\
F7 & 1.1827 & 0.7687 & 0.0617 & 0.8014 & 97.816 & 0.9199 \\
\hline
\end{tabular}

After undergoing the release models for all the formulations the in vitro drug release of the optimized formulations F4 and F5 were best explained by first order, as the plots showed the highest linearity with determination coefficient $\mathrm{R}^{2}=0.9902$ and $\mathrm{R}^{2}=0.9013$ respectively. The rate laws predicted by the different mechanisms of dissolution both alone and in combination, have been discussed by Higuchi. The equation in resemblance to the other rate law equations, predicts a first order dependence on the concentration gradient between the static liquid layer next to the solid surface and the bulk liquid. Noyes and Whitney explained their dissolution data using a concept similar to that used for the diffusion model. The data generated for all the formulations not fitted well to zero order kinetics and this model was not suitable to explain the rate kinetics for oral fast dissolving film formulations. The data was fitted with Higuchi equation which yielded almost linear plots with their high determination co-efficient $\mathrm{R}^{2}$ ranging from 0.926 to 0.991 for all formulations indicating that the mechanism of drug release was diffusion.

Summary and Conclusion: The main objective of the study was to formulate Zolpidem tartarate films as an alternative to Zolpidem sublingual tablet (INTERMEZZO) and evaluate the films. ODFs are prepared using three grades of polymers HPMC E5, GUAR GUM and SODIUM ALGINATE Compatibility of Zolpidem tartarate with polymers was confirmed by FT-IR studies. All the formulations were evaluated for their physical appearance, average weight, and thickness, folding endurance, disintegration time, tensile strength, percentage elongation, drug content, content uniformity and in vitro drug dissolution studies. Uniformity in the weights, thickness of the films indicated accuracy of dose in all formulations. F4 formulation showed least thickness of $0.081 \pm 0.02 \mathrm{~mm}$. tensile strength and folding endurance was increased with increase in the concentration of the polymer due to the increase in the elasticity nature of the polymer. Percentage drug content studies showed that the drug is uniformly distributed within the films. All the films acceptable content uniformity range as per 
USP. Disintegration and in vitro dissolution studies proved that the films instantly get wet because of great surface area and the films disintegrate and release the drug by the mechanism of film forming polymer erosion. From the drug dissolution characteristics, formulations formulated by HPMC E 5 have shown faster dissolution than GUAR GUM formulations (F5, F6 and F7). F4 formulation exhibited required Disintegration time of $49 \pm 2$ seconds, acceptable content uniformity, and Tensile strength of $1.88 \pm 0.02 \mathrm{~N} / \mathrm{cm}^{2}$ and in vitro dissolution of $99.98 \%$ within 15 minutes. From the result, it was concluded that the fast dissolving films of Zolpidem tartarate can be made by solvent casting technique with enhanced dissolution rate and taste masking by using suitable combination of sweeteners, flavors and citric acid. The final composition optimized was drug to Guar Gum ratio of 1:1, plasticizer concentration of $15 \% \mathrm{w} / \mathrm{w}$ of polymer. The film had acceptable physical properties, assay and uniformity values and in vitro dissolution within 2 minutes.

\section{References}

Bogan, R. K. (2008). Treatment options for insomnia-Pharmacodynamic of Zolpidem extended release to benefit next-day performance. Postgrad Med, 120, 161-171. https://doi.org/10.3810/pgm.2008.09.1916

Kai, B. L., Yvonne, T. F. T., \& Kok, K. P. (2012). Characterization of Oral Disintegrating Film Containing Donepezil for Alzheimer Disease. AAPS, 13(1), 134-142.

Lemmer, B. (2007). The sleep-wake cycle and sleeping pills. Physiol. Behav, 90(2-3), 285-293. https://doi.org/10.1016/j.physbeh.2006.09.006

Rosenberg, R. P. (2006). Sleep maintenance insomnia: Strengths and weaknesses of current pharmacologic therapies. Ann Clin Psychiatry, 18(1), 49-56. https://doi.org/10.1080/10401230500464711

Staner, L., Eriksson, M., Cornette, F., Santoro, F., Muscat, N., Luthinger, R., \& Roth, T. (n.d.). Sublingual Zolpidem is more effective than Zolpidem in initiating early onset of sleeping the post-nap model of transient insomnia: Apolysomnographic study. Sleep Medicine, 10, 616-620.

Suresh, B., Halloran, D., \& James, L. (2006). Quick dissolving films: A novel approach to drug delivery. Drug.dev.tech, 2006, 1-7.

Ulrike, V., \& Paolo, G. (2006). Rapid film: Oral thin films (OTF) as an innovative drug delivery systems and dosage forms. Drug delivery report spring/summer, 64-67.

Vondrak, B. (2008). Barnhart, Scott. Dissolvable Films: Dissolvable Films for Flex Product Format in Drug Delivery. Pharmatech, 2008, 1-5. 